Voix et Images

\title{
Un classique, un terroiriste et deux sacrés lurons
}

\section{André Brochu}

Volume 29, numéro 3 (87), printemps 2004

France Daigle

URI : https://id.erudit.org/iderudit/009229ar

DOI : https://doi.org/10.7202/009229ar

Aller au sommaire du numéro

\section{Éditeur(s)}

Université du Québec à Montréal

\section{ISSN}

0318-9201 (imprimé)

1705-933X (numérique)

Découvrir la revue

Citer ce compte rendu

Brochu, A. (2004). Compte rendu de [Un classique, un terroiriste et deux sacrés lurons]. Voix et Images, 29(3), 161-165. https://doi.org/10.7202/009229ar d'utilisation que vous pouvez consulter en ligne.

https://apropos.erudit.org/fr/usagers/politique-dutilisation/ 


\author{
P O ÉS I E \\ Un classique, un terroiriste et deux sacrés lurons \\ $+++$
}

ANDRÉ BROCHU

Université de Montréal

Les amateurs de curiosités littéraires et artistiques se réjouiront de la réédition du premier ouvrage en vers paru au Québec, Épitres, satires, chansons, épigrammes et autres pièces de vers ${ }^{1}$ de Michel Bibaud (1830), et du livre-album que Gaëtan Dostie et JeanGuy Paquin consacrent à Albert Ferland ${ }^{2}$, poète, illustrateur et éditeur. Les esprits plus strictement littéraires feront plutôt porter leur choix sur Jean-Aubert Loranger ${ }^{3}$ et Louis Dantin $^{4}-$ si, bien entendu, ils n'ont pas acheté une réédition à peine moins récente: j'y reviendrai.

\title{
UN SATIRISTE À MONTRÉAL
}

Le sacré luron, à vrai dire, ce serait plutôt Michel Bibaud qui prend sa grosse voix et feint de fustiger les travers de ses contemporains sur un air connu, celui des Satires de Boileau. Bibaud est l'un des premiers, parmi nos écrivains, à faire de la littérature en s'appuyant sur les modèles français, comme si l'originalité n'avait pas d'importance ou mieux, comme si écrire consistait très exactement à se parer des plumes du paon. La leçon ne sera pas perdue. D'ailleurs, le romantisme n'a pas encore abordé les rives du Saint-Laurent et n'a donc pas infecté nos rares beaux esprits, avec ses exigences d'innovation.

La première «Satire» commence ainsi: «Heureux qui dans ses vers sait, d'une voix tonnante,/Effrayer le méchant, le glacer d'épouvante» (25). Dans son Art poétique, Boileau, sans doute connu par cœur de tous les collégiens de l'époque, écrivait plutôt: «Heureux qui, dans ses vers, sait d'une voix légère/Passer du grave au doux, du plaisant au sévère!» (I, 75-76). Le plagiat est patent, mais difficile à interpréter. De l'ironie s'y mêle peut-être. On pourra toujours admirer l'écart que marque notre sympathique poète local entre sa tonitruante et militante vertu et le culte de la raison et de la nuance propre à Boileau. Bibaud est un classique, certes, et il fait comme son maître l'éloge de la raison, mais il s'érige surtout en imprécateur et en prédicateur. Boileau aussi entend corriger les

$$
+++
$$

1 Michel Bibaud, Épîtres, satires, chansons, épigrammes et autres pièces de vers, présentation de Bernard Pozier, Montréal, Les Herbes rouges, coll. "Five O'Clock», 2003, 230 p. 2 Albert Ferland, 1972-1943. Du pays de Canard Blanc Wâbininicib au plateau Mont-Royal, édition préparée par Gaëtan Dostie et Jean-Guy Paquin, Montpellier, Écrits des Hautes-Terres, coll. «Outaouais», 2003, 272 p. 3 Jean-Aubert Loranger, Les atmosphères suivi de Poëmes, postface de Luc Bonenfant, Québec, Nota bene, 2004, 166 p. 4 Louis Dantin, La triste histoire de Li-Hung Fong et autres poèmes, choix et présentation de François Hébert, Montréal, Les Herbes rouges, coll. «Five O'Clock», 2003,124 p. 
vices - le moralisme se porte bien, à la cour de Louis XIV -, mais il le fait avec esprit. L'esprit n'est pas le fort du Canadien. Ses écrits rappellent ceux d'un collégien, où la bonne volonté le dispute à la maladresse.

Autre exemple de plagiat, là encore au début d'une «Satire», la quatrième, "Contre l'ignorance». Bibaud va pousser la coquinerie jusqu'à mettre en italique quelques mots qu'il identifie en note comme un «hémistiche de Boileau»:

Mon étoile, en naissant, ne m'a point fait poète;

Et je crains que du ciel l'influence secrète

Ne vienne point exprès d'un beau feu m'animer.

Or, c'est tout le premier vers et la quasi totalité du deuxième qui reprennent le début de L'art poétique:

\author{
C'est en vain qu'au Parnasse un téméraire auteur \\ Pense de l'art des vers atteindre la hauteur: \\ S'il ne sent point du ciel l'influence secrète, \\ Si son astre en naissant ne l'a formé poète, \\ Dans son génie étroit il est toujours captif (I, 1-5; je souligne).
}

On pourrait poursuivre longuement la chasse aux emprunts. Elle montre Bibaud soucieux de s'accaparer magiquement une inspiration qui équivaut au magistère par excellence. Se faire Boileau, c'est incarner sinon la poésie - nos deux B prétendent modestement n'être que des rimeurs - , du moins la littérature dans son exercice le plus flatteur, puisqu'il se confond avec celui de redresseur des torts de chacun. Corriger, fouetter, flageller, porter des coups, donner du bâton ou de la férule, faire main basse sur les vilains, frapper d'estoc et de taille, effrayer les méchants, ce riche lexique réaffirme sans cesse un sadisme trop excessif pour n'être pas faux. Bibaud s'amuse, sans toutefois trouver le moyen d'amuser son lecteur.

Mais au moins, chaussé des bottes de Boileau, parfois de La Fontaine ou de La Bruyère, il fait quelques lieues en écriture, rimaillant et tonnant. Il ouvre ainsi une première voie à la littérature au sein de nos arpents de neige, et une tonalité composée de bonne humeur, de simplicité, de naturel perce par-ci par-là le fatras des emprunts ou des plates réprobations ${ }^{5}$.

Bernard Pozier, qui présente l'œuvre, mentionne La Fontaine, mais ne dit pas un mot de l'inspirateur en chef. Il cherche surtout les échos de Bibaud dans une postérité qui va jusqu'à l'Hexagone et aux récents poètes chantres de l'Amérique, voire aux formalistes (Bibaud a écrit un poème avec des rimes en ec, ce qui n'en fait pourtant pas le précurseur d'André Gervais!). C'est là ramener la poésie à quelques aspects très élémentaires, surtout thématiques (au sens large du mot), en faisant fi de l'écriture.

\title{
L'ORPHÉE DES OUAOUARONS
}

Les «exotiques» revivent facilement dans les anthologies et les rééditions, ces dernières années. On ne peut en dire autant de leurs adversaires, les régionalistes, qui ont eu la vie

5 Par exemple, contre le tabac... 
plus facile en leur temps et qui semblent maintenant condamnés à l'oubli - ou presque. La parution d'un livre-album (c'est-à-dire d'un livre de format normal où la reproduction photographique l'emporte sur le texte composé) vient rappeler le souvenir d'Albert Ferland.

Malgré la relative abondance des poèmes tirés des recueils ou d'autres publications de l'auteur et des inédits, on ne peut parler d'un choix de textes en bonne et due forme car aucun plan ne semble présider à leur réunion. Gaëtan Dostie et Jean-Guy Paquin sont des zélateurs de Ferland - Dostie l'est à sa façon bien particulière qui est celle du collectionneur. Ils se passionnent autant pour son travail d'éditeur et d'illustrateur que d'écrivain, et la qualité proprement littéraire des poèmes leur importe peu. Il leur suffit de reprendre l'éloge de la sœur du grand homme, qui lui attribuait l'inestimable mérite d'avoir le premier chanté les ouaouarons, les pissenlits, la chicorée et autres merveilles de chez nous. Assez curieusement, notre contemporain, Jean-Pierre Issenhuth, a priori peu suspect de complaisance pour le terroir, raffole des poèmes sur de semblables sujets (mais traités, bien entendu, de façon supérieure) ${ }^{6}$.

Malgré le côté convenu des sujets abordés, Ferland n'est pas dépourvu du sens poétique et se retrouve souvent proche des «exotiques», notamment René Chopin, ou de poètes moins enrégimentés comme Albert Lozeau; lesquels, ne l'oublions pas, ont eux aussi chanté la nature. Ferland ne célèbre pas que les ouaouarons, et s'il évoque la «Poésie des Feuilles» en termes d'abord assez banals ( Splendeur des bois de mon pays,/Vous toutes les feuilles que j'aime...»), il aboutit à des notations plus originales:

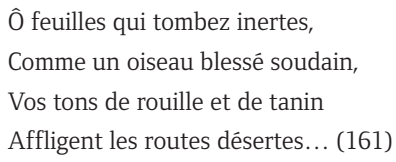

C'est le troisième vers, ici, qui relève tout et sauve le quatrain.

Malgré la présence d'authentiques réussites, on s'étonne du nombre élevé de vers boiteux et de certaines fautes de goût. Le premier quatrain d' «Impromptu matinal», dont la sensibilité rappelle celle des symbolistes, aboutit à un mot malencontreux:

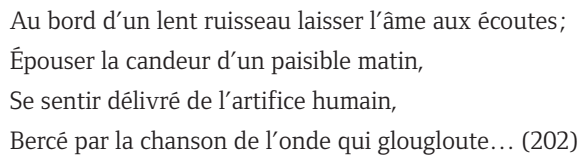

Après l'«artifice humain", qui connote une authentique hauteur d'âme ou d'esprit, les glouglous déconcentrent... Cette chute dans le trivial trouve peut-être sa justification dans un schème imaginaire propre à l'auteur puisqu'on en rencontre l'équivalent dans un poème connu, "Le rêve du héron bleu», dont les perspectives éthérées cèdent finalement la place au constat brutal. Tel est le cas d'abord dans le premier quatrain, d'une grande force évocatrice, dont les images mettent l'accent sur la dimension de verticalité et l'absence au réel, apparente seulement:

\footnotetext{
6 Ma chronique pour le no 86 est entièrement consacrée au récent recueil de chroniques de J.-P. Issenhuth.
} 
Dès l'aube un héron bleu s'est figé comme un jonc

Sur le bord du lac vierge où son image plonge.

On le dirait surpris par le philtre d'un songe,

Évadé du réel, béat sur son pied long. (175)

Le héron "figé comme un jonc», formulation qui insiste sur l'aspect filiforme de l'échassier et métaphore descendante puisqu'on passe de la vie animale à la vie végétale, est à la fois le support des notations de rêve qui se développent dans les vers suivants et l'annonce discrète d'une note plus réaliste, que suggère le mot «béat», aux connotations péjoratives. Le contraste entre l'idéal et la réalité ira s'accentuant jusqu'à la chute du poème, qui le ramasse en une formule grinçante: «Et, svelte dans l'aurore, il incarne la Faim». La rhétorique symboliste sert ici le regard naturaliste. Ce qui donne à penser que certains régionalistes n'étaient pas si loin des «exotiques».

Un inventaire rigoureux des richesses littéraires de l'œuvre de Ferland serait le bienvenu, et il faut saluer la sympathique tentative de Dostie et de Paquin pour nous redonner accès à cet écrivain oublié. Au fait, pourquoi la collection «Five O'Clock» n'accueillerait-elle pas une anthologie des régionalistes?

\section{DES REVENANTS VITE REVENUS}

Je parlerai rapidement des livres de Loranger et de Dantin pour la bonne raison que ces deux auteurs ont été réédités récemment, par les soins d'autres présentateurs et dans $\mathrm{d}^{\prime}$ autres maisons d'édition ${ }^{7}$. Or le travail des éditeurs avait été fort convenable. Sans doute, la présentation matérielle de Loranger aux éditions Nota bene est-elle plus que remarquable et la postface de Luc Bonenfant est d'une belle pénétration sur le plan thématique; on peut louanger aussi l'appareil critique dont François Hébert accompagne le choix de poèmes de Dantin ${ }^{8}$. Il n'en reste pas moins que le bassin de lecteurs pour de telles publications est exigu, et qu'on devrait éviter de saturer le marché.

Dans sa réédition de Loranger, Dominique Robert avait laissé de côté deux textes en prose des Atmosphères: un conte et un poème connu, «Le passeur». Il est heureux que Nota bene les ait repris, surtout ce dernier. Le travail de l'écriture y est particulièrement poussé. On y trouve ce beau côté tordu ${ }^{9}$ qui donne aux textes de Loranger un air parfaitement étrange dans la représentation du banal, du quotidien. L'usage du verbe «être» est étonnant dans des passages comme celui-ci, qui décrit la maladie du vieillard éponyme: «Il fut le dos malade qui refuse aux bras le muscle dont il est la racine; il fut la fissure; il fut l'attente de la mort devant tout cela qui est la vie...» (26). Autant la simple copule sert à formuler les relations les plus complexes, autant d'autres verbes, plus concrets, apportent leur concours pour étonner encore davantage.

Par exemple, dans ce passage qui rend la réflexion du passeur, «des reins, ça ne servait à rien, sinon à faire souffrir, quand on les attrape» (20). Attraper des reins? L'explication vient plus bas, en deux étapes. La première est assez abstraite: «Son mal et ses reins s'identifièrent donc en passant par sa connaissance. Ils furent une partie

\section{$+++$}

7 Jean Aubert Loranger, Signets et autres poèmes, choix et présentation de Dominique Robert, Montréal, Les Herbes rouges, coll. «Five O'Clock», 2001, 116 p.; Louis Dantin, L'abîme hospitalier, choix et présentation d’Éric Roberge, Trois-Rivières, Écrits des Forges, 2000, 134 p. 8 Sur Dantin, François Hêbert est aussi l'auteur d'une très belle étude, «L'hostie de Dantin", Les Écrits, n 97, décembre 1999, p. 9-30. 9 Dans une récente chronique (Voix et Images, nº 82, automne 2002, p. 171-174), j'ai parlé de "génie maladroit» à propos du jeune auteur des Atmosphères et des Poëmes. 
douloureuse à son corps.» En termes plus convenus, il eut mal aux reins et prit connaissance de la douleur. Puis: «Puisque ses reins étaient le mal à son corps, il avait donc attrapé les reins.» Attraper du mal, quand les reins sont le mal (ne font qu'un avec lui), $c^{\prime}$ est attraper les reins. Et ainsi, dans la vie d'un pauvre hère qui appartient au plus petit dénominateur commun de la société, le corps devient chose métaphysique, passe (ne s'agit-il pas d'un passeur, d'un trait d'union entre deux rives?) de la matière à l'esprit, se transmue en idée.

Loranger fait acte de modernité en se montrant proche de la poésie française de son temps, notamment de l'unanimisme de Romains et de Vildrac. Cela ne l'empêchera pas de pratiquer le régionalisme dans ses contes, une fois qu'il aura fini de jeter sa gourme. Albert Ferland n'était pas si éloigné des symbolistes, et Loranger, déjà à l'époque du «Passeur», aurait pu signer certains écrits du terroir.

Quant à Dantin ${ }^{10}$, il était un homme d'une grande intelligence et d'une grande culture. De son propre aveu, il n'a pas réussi à trouver sa voix propre en poésie, mais ses écrits, en vers ou en prose, ne manquent jamais de rigueur ni d'intérêt. Son sens social, plus développé que celui de ses contemporains captifs de l'aliénation religieuse ou du libéralisme à l'américaine, va curieusement s'exprimer dans des «chansons» qui, comme les poèmes de Jean Narrache, mettent en avant le dépossédé, dans un langage mimétique, plein des élisions de la langue parlée. C'est le cas pour «La triste histoire de LiHung Fong» et quelques autres textes. Ce qui étonne, c'est que ce «joual» avant la lettre s'applique à un personnage exotique. En fait, il ne s'agit pas de joual mais d'une langue populaire à la Jehan Rictus, qui ne reflète pas une misère ethnique en particulier.

Mais on trouve aussi d'autres inspirations, la trajectoire du poète - écrit François Hébert - allant «du spirituel à l'intellectuel et au sensuel...» (119). J'en ai parlé ailleurs.

Dantin et Loranger sont deux êtres à part, qui ont un pied dans la grande littérature (représentée essentiellement par Nelligan) et l'autre dans les limbes de l'inachèvement. En ce sens, ce sont de sacrés lurons, hommes qui vont «au hasard, sans direction précise», dit l'étymologie. À distinguer de l'autre sens du mot «luron»: «diseur de sornettes», qui s'applique plutôt à Michel Bibaud si on le lit au premier degré... 